\title{
NFAT in Pancreatic Carcinogenesis
}

\author{
Elisabeth Hessmann, Volker Ellenrieder and Alexander Koenig \\ Department of Gastroenterology II, University Medical Center Goettingen, Germany
}

*Corresponding author: Volker Ellenrieder, Department of Gastroenterology II, University Medical Center Goettingen, Robert Koch Strasse 40, 37075 Goettingen, Germany, Tel: +49 55139-63 63; Fax: +49 551 39-69 21; E-mail: volker.ellenrieder@med.uni-goettingen.de

Rec date: Jul 12, 2014, Acc date: Nov 21, 2014, Pub date: Nov 30, 2014

Copyright: (C) 2014 Hessmann E, et al. This is an open-access article distributed under the terms of the Creative Commons Attribution License, which permits unrestricted use, distribution, and reproduction in any medium, provided the original author and source are credited.

\begin{abstract}
Pancreatic Ductal Adenocarcinoma (PDAC) belongs to the most aggressive malignancies among solid tumors. The increasing incidence of PDAC and its dismal prognosis with a five-years overall survival rate of less than five percent characterize PDAC as one of the most clinically challenging diseases among all malignancies. In recent years, increasing knowledge about the molecular pathogenesis of PDAC has shown that mutational activation of oncogenic Kras represents the defining event in pancreatic cancer initiation, but full neoplastic pancreatic cancer progression requires dysregulation of additional pathways. In this review we summarize and discuss the impact of inflammation-induced transcription of the Nuclear Factor of Activates T-cell (NFAT)-family in pancreatic carcinogenesis.
\end{abstract}

Keywords: NFAT; Pancreatic cancer; Transcription

\section{Introduction}

The current model of pancreatic carcinogenesis describes a stepwise transformation of healthy exocrine cells into highly invasive pancreatic cancer cells [1,2]. Despite the ductal morphology lineage tracing studies revealed that PDAC tumors arise from the acinar compartment. In fact, early genetic alterations in acinar cells induce a transdifferentiation program termed Acinar-To-Ductal Metaplasia $(\mathrm{ADM})$ in which acinar cells adopt a duct-like structure and loose expression of acinar-cell differentiation markers [3]. Depending on the molecular context, ADM lesions then convert to pancreatic cancer Precursor Lesions (PanIN) with increasing degrees of dysplasia and can progress to invasively growing pancreatic cancer $[4,5]$. The morphological changes during pancreatic carcinogenesis are accompanied by a recurrent pattern of genetic and epigenetic alterations; the earliest and most prevalent of which is oncogenic activation of Kras [1]. Kras-proteins comprise a family of signaltransducing GTPases involved in regulation of vital cellular functions including differentiation, proliferation, survival and apoptosis. Mutational activation of Kras is found in early tumor stages and in virtually all invasively growing pancreatic tumors [6]. The impact of Kras mutation for pancreatic carcinogenesis has been elegantly demonstrated in Genetically Engineered Mouse Models (GEMM) with pancreas-specific constitutive activation of the enzyme (KrasG12D) [5]. However, studies in mice and humans have revealed that Kras mutation alone is not sufficient for full neoplastic progression and rather induces a permanent cell growth arrest called senescence. Therefore, additional genetic and epigenetic events [7] such as deficiency of prominent tumor-suppressor genes, e.g. p53 and p16Ink4a, are required to bypass oncogene-induced senescence and apoptosis and to stimulate tumor promotion and progression $[7,8]$. Moreover, signals from an oncogenic tumor environment are involved in pancreatic tumor development and progression. This type of prooncogenic microenvironment can be provided by sustained organ inflammation, e.g. chronic pancreatitis, which constitutes the most relevant risk factor for PDAC development in human $[9,10]$. Chronic pancreatitis supports the initiation and progression of this malignancy by direct modification of gene expression networks in pancreatic epithelial cells, specifically through activation of inflammatory transcription factors.

One of these oncogenic transcription factors is the calcineurinresponsive Nuclear Factor Of Activated T cells (NFAT) family, originally identified as a group of inducible nuclear proteins, which regulate transcription during $T$ lymphocyte activation [11]. However, a multitude of studies established that NFAT expression and function is not restricted to the immune system, but participates in gene regulation in multiple tissues [12]. The different NFAT isoforms are functional in tumor cells as well as in multiple compartments of the tumor environment and ectopic activation of NFAT family members is now recognized as an important aspect for oncogenic transformation in several human malignancies, most notably in pancreatic cancer $[13,14]$. In this issue we describe the regulation and activation of NFAT members in pancreatic acinar cells and review the relevance of NFAT-mediated transcription in the sequential steps of pancreatic carcinogenesis.

\section{NFAT Regulation and NFAT-Mediated Transcription in Pancreatic Carcinogenesis}

The family of NFAT transcription factors comprises four Calcium/ Calcineurin-responsive proteins (NFATc1-c4) [15]. In resting cells, NFAT proteins reside in the cytoplasm in a highly phosphorylated and inactive state [16]. Inflammatory cytokine binding to corresponding receptors causes increased intracellular calcium levels, followed by activation of the phosphatase calcineurin which targets and dephosphorylates NFAT proteins in the cytosol [17]. Subsequently, dephosphorylated NFAT shuttles into the nucleus, where it becomes ubiquitinated for HDM2-dependent proteasomal degradation or protected by GSK3ß-mediated phosphorylation (Figure 1) [18]. Upon stabilization, the transcription factor binds to its consensus sequence GGAAA within target gene promoters and enhancers to promote or repress transcription. NFAT-dependent transcription requires functional and biochemical cooperation with partner proteins that 
determine target gene selection and the mode of transcription (repression versus activation) by NFAT proteins [14]. Thus, the nuclear composition and activation status of NFAT-partner complexes highly contributes to the broad spectrum of cellular functions that are attributed to the NFAT family.

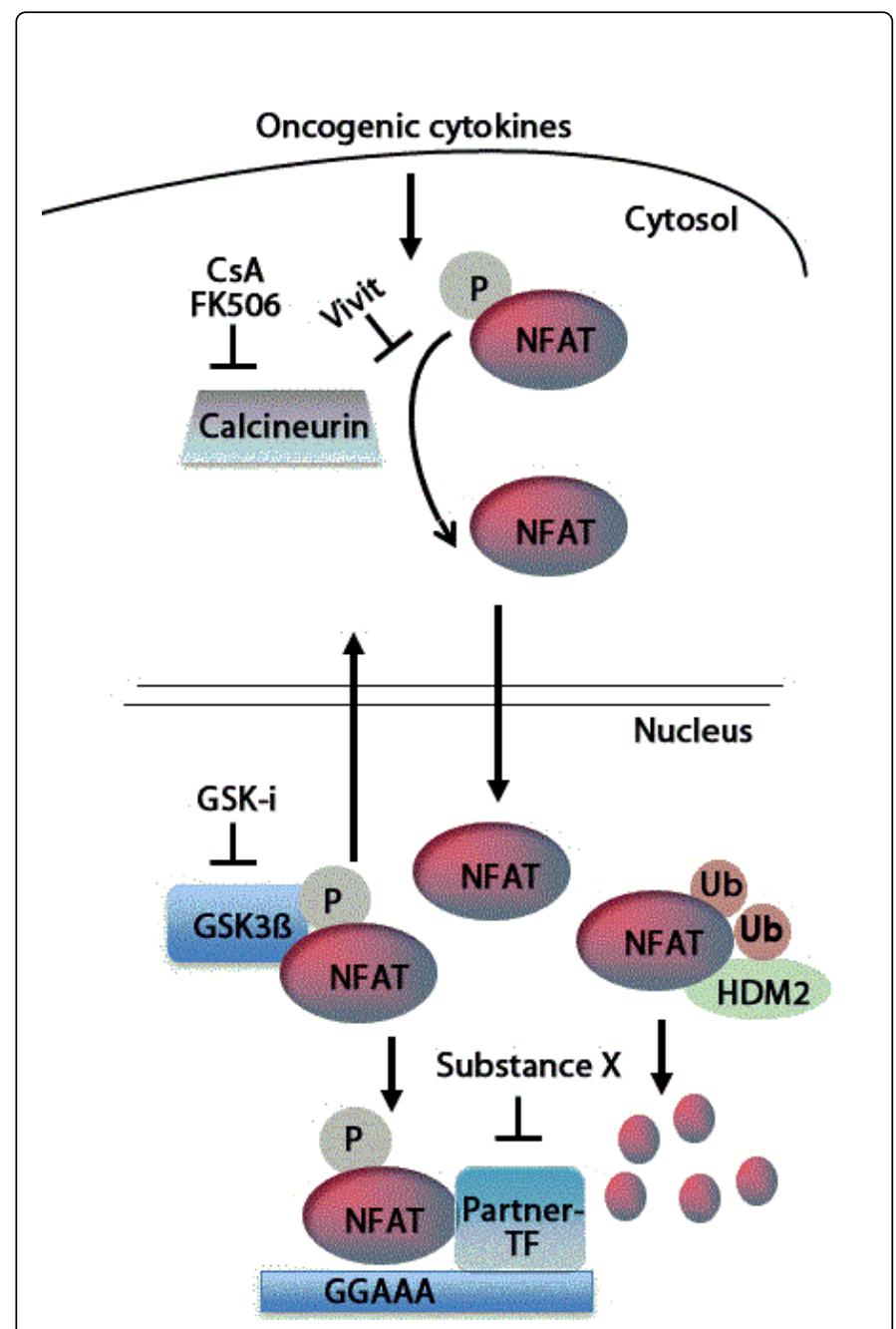

Figure 1: NFAT signaling in pancreatic cancer cells: Inactive cytosolic NFAT proteins are dephosphorylated by calcineurin in response to oncogenic cytokines, and elevated intracellular $\mathrm{Ca} 2+$ concentrations, allowing their nuclear translocation. In the nucleus, active NFAT proteins either become ubiquitinated for proteosomal degradation or are stabilized by GSK3ß-dependent phosphorylation, thus allowing interaction with partner transcription factors on distinct target genes.

NFAT transcription factors promote pancreatic carcinogenesis via distinct transcriptional programs leading to increased proliferation and growth, inhibition of critical fail-safe mechanisms and interference with key tumor-suppressor functions. In addition, NFAT proteins contribute to tumor cell escape from the adaptive immune system and eventually drive tumor cell dissemination via induction of Epithelial Mesenchymal Transition (EMT) and acquisition of stem cell features, all together characterizing NFAT transcription factors as powerful oncogenes in pancreatic cancer. Recent studies on NFATmediated gene regulation further extended the repertoire of oncogenic
NFAT-activities in PDAC and identified additional key functions of the family in the earliest phases of pancreatic cancer initiation, namely the transcriptional induction of acinar-to-ductal metaplasia (ADM).

\section{Implication of NFATc1 in pancreatic cancer initiation}

The cellular origin of PDAC has been a controversial topic for many decades. In recent years, increasing evidence indicates that PanIN formation and invasive PDAC originate from differentiated acinar cells via a trans-differentiation program termed acinar-ductal metaplasia. Inflammation-linked activation of the Epidermal Growth Factor Receptor (EGFR) in patients suffering from chronic pancreatitis as well as in early pancreatic cancer stages suggests an involvement of EGFR mediated processes in ADM [19]. However, though accumulating evidence from human and murine studies support an essential role of EGFR signaling activation in inflammation-driven metaplasia and pancreatic cancer initiation $[3,20]$, the molecular mechanisms linking EGFR with ADM remained elusive.

NFAT transcription factors participate in the regulation of genes influencing the development and differentiation of multiple tissues, including chondrocyte differentiation, myogenesis and the development of the cardiovascular system [21,22]. In line with this, recent findings of our group describe a critical involvement of NFATc1 in acinar transdifferentiation. EGFR mediated ADM involves complex formation of NFATc1 and AP1 proteins in the metaplastic epithelial cell and requires NFATc1 dependent transcriptional activation of target genes crucial for ADM formation. Pharmacological or genetic inhibition of NFATc1 in GEMM with a conditional knockout of the transcription factor KrasG12D;NFATc1fl/fl;pdx1-Cre significantly impaired acinar-to-ductal transdifferentiation. Thus our results link EGFR-NFATc1 signaling with inflammation-induced pancreatic cancer initiation and open new doors for preventive therapeutic options for high-risk pancreatitis patients.

\section{NFAT in proliferation and pancreatic cancer growth}

Following their initial discovery, a multitude of studies quickly established that NFAT participates in the regulation of genes influencing differentiation and cell growth. One of the first studies implicating NFAT transcription factors in proliferation and growth was performed in fibroblasts, where constitutive activation of NFATc1 induced colony formation and transformation [23]. In pancreatic tumor cells, proliferation and anchorage-independent growth is - at least in part - dependent on calcineurin activity and nuclear translocation of NFATc1 and NFATc2 [24], underlining the oncogenic impact of both transcription factors in pancreatic cancer. An increased cell-cycle re-entry and the acceleration of G1-S phase transition are appreciated as hallmarks of increased tumor cell proliferation $[25,26]$. This process is dependent on rapid and sustained induction of immediate early transcriptions factors (ITF) [27]. Proto-oncogenic cMyc was identified as a master ITF in G1-S cell-cycle progression in various tumor entities, including PDAC [24]. In untransformed acinar cells, c-Myc activation is repressed by Transforming Growth Factor $B$ (TGFß) - signaling. C-Myc repression by TGFß requires activation of a Smad3/4 repressor complex that binds to the TGFß-inhibitory element (TIE) within the proximal c-Myc promoter, resulting in transcriptional silencing of the gene [28]. However, during pancreatic carcinogenesis, many tumor cells change their transcriptional responsiveness to TGFß and become resistant to its growth-inhibiting 
effects due to functional or mutational inactivation of the TGFß-Smad pathway [29].

1.

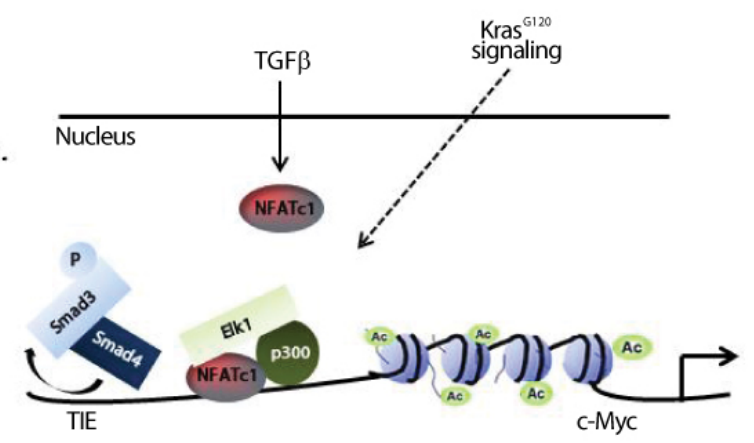

2.

$$
\text { Nucleus }
$$

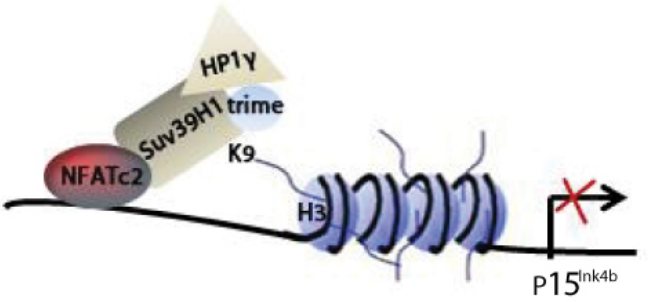

3.

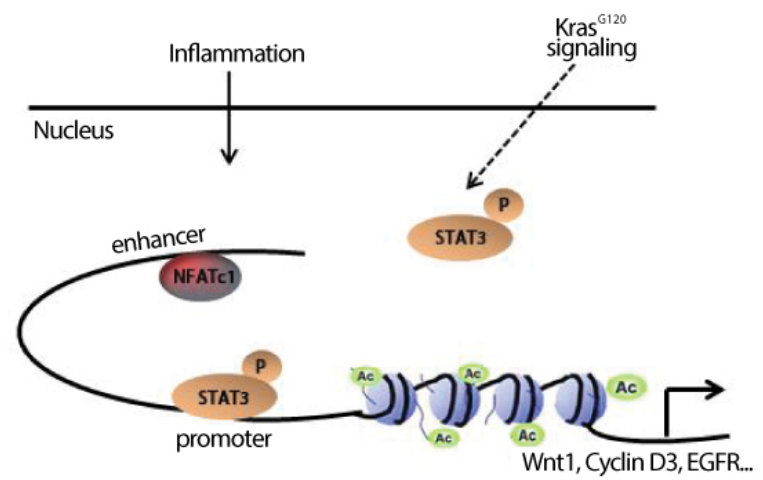

Figure 2: NFAT controls transcription in pancreatic cancer: 1. TGFß induces NFATc1 in pancreatic carcinogenesis for c-Myc activation: NFAT binding to the proximal c-Myc promoter leads to displacement of the Smad $3 / 4$ complex from the TIE and involves recruitment of p300 actelytransferases and Elk1 to induce a chromatin structure permissive for c-Myc transcription. TIE: TGFß-inhibitory element. 2. NFATc2 binding to the proximal p15Ink4b promoter results in sequential heterochromatin formation by recruitment of Suv39H1 and trimethylation of $\mathrm{H} 3 \mathrm{~K} 9$ to silence p15Ink4b - mediated tumor suppression. 3. NFATc1/ STAT3 communication: NFATc1 and STAT3 activate jointly regulated target genes via loop formation between promoters and enhancers in pancreatic carcinogenesis.
In fact, more than $50 \%$ of human pancreatic cancers harbour inactivating mutations in the Smad4 (DPC4) tumor suppressor gene [30] Upon loss of Smad signalling, TGFß can control gene regulation in pancreatic cancer cells through Smad-independent pathways [17], including activation of PI3K and MAPK signaling [29], to activate non-Smad transcription factors. One such factor is NFAT that is highly induced upon TGFß stimulation, and accumulates in the nucleus to govern gene expression. Importantly, NFAT proteins can displace pre-existing Smad3 repressor complexes from the c-Myc TIE element and induce p300-dependent histone acetylation thus rendering the promoter transcriptionally active. Hyperacetylation of the c-Myc promoter is required for the recruitment of the Ets-like gene 1 (ELK-1), a protein that signals downstream of Kras and is crucial for maximal activation of c-Myc (Figure 2) [27]. While NFATmediated transcriptional activation of c-Myc increases G1-S phase transition and results in accelerated tumor cell growth in vivo and in vitro, genetic or pharmacological depletion of NFAT proteins restores TGFß growth suppression in pancreatic cancer cells and impairs c-Myc expression [27,29], thus emphasizing the functional significance of this pathway in pancreatic cancer proliferation.

The oncogenic potential of NFATc1 in pancreatic carcinogenesis and tumor cell proliferation is mirrored in GEMM with constitutive activation of NFATc1 [31]. While mice bearing an acinar cell specific singular constitutive activation of NFATc1 failed to develop advanced PanIN lesions within the observation span of one year, combined pancreas-specific activation of NFATc1 and Kras (KrasG12D;NFATc1fl/fl;pdx1-Cre), a situation found in $70 \%$ of human PDAC diseases, resulted in a dramatic acceleration of pancreatic carcinogenesis and led to an extremely shortened survival compared to KrasG12D;pdx1-Cre animals. Further resembling human PDAC, KrasG12D;NFATc1fl/fl;pdx1-Cre mice mice developed severe cachexia and abdominal distension due to sanguineous ascites and bile duct obstruction. Upon necropsy, the pancreata of KrasG12D;NFATc1 mice displayed enlarged pancreatic tumor masses with both cystic and solid regions and regularly showed liver metastases. This pancreatic cancer GEMM which nicely recapitulates the human disease, hints on synergistic effects of Kras-signaling and NFAT mediated transcription. Recent studies established that NFATcl partners with the Krasdownstream target signal transducer and activator of transcription-3 (STAT3) [31]. In depth-going investigations including ChIP-Seq combined with genome-wide expression analysis revealed that NFATc1- STAT3 interaction comprises the formation of promoterenhancer communications on jointly regulated target genes involved in proliferation and growth, including cyclines, Wnt-famliy members and EGFR (Figure 2).

Summarily, intensive studies in human and murine pancreatic cancer models describe a crucial function of NFAT transcription as a promoter of proliferation and growth in Kras-driven pancreatic carcinogenesis. Moreover, NFAT family members maintain pancreatic tumor cell proliferation and pancreatic cancer progression via inhibition of tumor-suppressive signals.

\section{NFAT Mediated Disruption of Tumor Suppression in Pancreatic Carcinogenesis}

Counteracting transformation and growth, cellular senescence, a permanent cell growth arrest, is recognized as one of the most critical fail-safe mechanisms impeding pancreatic carcinogenesis [32]. A major course of this permanent cell-cycle arrest was found in telomeres which shorten progressively during devision of normal cells 
and force the cell to become senescent, when telomeres erode down below a threshold length [33,34]. Importantly, senescence can also be found in the absence of any detectable telomere shortening, for example as a response to oncogene activation. Oncogene induced senescence (OIS) has emerged as a powerful tumor suppressive mechanism protecting cells from unrestrained proliferation imposed by oncogenic signaling [35]. In response to oncogenic activation of Kras in the pancreas the Ink4 family of cell cycle inhibitors (p15Ink4b,p16Ink4a and p14Arf) is highly induced and blocks cell cycle progression by inhibition of cyclines and their kinases [36,37].

Consistent with their central role in pancreatic tumor suppression, inactivation of p15Ink4b and its close relatives occurs in over $90 \%$ of PDACs [26,36]. Additional to mutational or deletion-caused inactivation of the cell-cycle inhibitors, silencing of tumor suppressor genes also occurs via epigenetic events. For example, NFATc2 targets p15Ink4b for inducible and sequential heterochromatin formation. NFATc2 binding to its putative binding site on the proximal p15Ink4b promoter leads to recruitment of the histone methyltransferase Suv39H1. Local trimethylation of lysine 9 on histone 3 (H3K9trime) allows docking of heterochromatin protein $1 \mathrm{y}$ (HP1y) which results in stabilization of the protein complex on the p15Ink4b promoter. Inactivation of NFATc2 disrupts the repressor complex and results in restoration of p15Ink4b expression and tumor-suppressive function (Figure 2) [37].

Additionally to senescence, NFATc2 activation downstream of Wnt5a impedes pancreatic apoptosis and thus promotes drug resistance and tumor cell survival [38]. Moreover, ongoing studies facing pancreatic chemoresistance and metastasis describe antithetical NFATc1 and p53-signaling pathways in pancreatic cancer, suggesting that NFAT transcription might impede p53-induced apoptosis (V.E. personal communication).

Supplementary to senescence and apoptosis tumor suppressive signals extinguish from a physiological immune response that comprises recognition and elimination of transforming cells through CD4- and CD8-positive T-cells [39]. During pancreatic carcinogenesis this cancer-entity independent tumor-suppressive mechanism is impeded by activation of myeloid-derived-suppressor cells (MDSC) in the pancreatic tumor stroma, which insulate pancreatic cancer cells from T-cell mediated inactivation [40]. Preliminary data suggests an essential role of NFAT proteins in the transcriptional induction of a core of cytokines associated with MDSC recruitment and activation (unpublished data). Hence, NFAT does not only counteract tumor suppression in the transforming pancreatic epithelial cell itself, but also contributes to pancreatic cancer progression by conducting the composition of the pancreatic environment.

\section{Perspective}

NFAT-dependent oncogenic activity constitutes a frequent event in pancreatic carcinogenesis that occurs in all steps of PDAC development and progression. NFAT-mediated transcription is crucial for PDAC initiation in ADM as well as in PanIN-progression towards invasive adenocarcinoma. Exceeding pancreatic carcinogenesis, NFAT is also functional in maintenance of invasive PDAC and inhabits crucial functions in pancreatic de-differentiation and metastases (unpublished data). The manifoldness of NFAT mediated functions in pancreatic carcinogenesis is - at least in part - due to various partner proteins that combine Kras-dependent signals with NFAT-mediated transcription. As oncogenic activation of Kras, which occurs in nearly all patients suffering from PDAC, evades any pharmacological intervention, targeting of oncogenic pathways that cooperate with Kras signaling might be a promising strategy for new therapeutic approaches. Three structural unrelated inhibitors, Cyclosporin A (CsA), VIVIT and FK506 display potent Calcineurin/NFAT inhibitors and are widely used as immunosuppressive drugs in tissue transplantation [41]. All three inhibitors prevent nuclear translocation of NFAT by interfering with calcineurin or the calcineurin/NFAT interaction (Figure 1) [11]. As genetic depletion of NFATc1 in mice effectively decelerates pancreatic carcinogenesis [31], one could predict, that the aforementioned inhibitors demonstrate effective cancer therapeutics. Paradoxically, there is a significant increase in cancer incidence in patients on long-term immunosuppressive treatments [42]. This might be caused by the fact that these inhibitors do not specifically target calcineurin/NFAT signaling in the transforming cell, but also inhibit differentiation and activation of tumor cell attacking T-cells. Although our knowledge about NFAT mediated transcription in pancreatic carcinogenesis has been extended within the last years, specific targeting of NFAT in this disease remains challenging. There is a great need for drugs that selectively target tumor-specific NFAT functions, e.g. via modulation of NFAT metabolism or disruption of tumor promoting partner complexes.

Addressing NFAT protein turnover and metabolism, for instance through inhibition of NFATc1 stabilizing kinases (e.g. GSK3ß), might offer interesting new therapeutic strategies in vitro and in vivo (Figure 1) [18]. Furthermore, accumulating evidence hints on broad NFAT cooperation with chromatin-remodeling proteins as Suv39H1, p300 and EZH2 [37,43-45] that demonstrate promising targets for new therapeutic approaches. Further investigations of NFAT-associated epigenetic mechanisms in pancreatic carcinogenesis will certainly extent the spectrum of pharmacological interference in this disease.

\section{Acknowledgments}

This work was supported by the DFG (V.E.: KFO210, SFB-TR17) and the German Cancer Research Foundation (to V.E.: N ${ }^{\circ}-109423$ and A.K.: "Mildred Scheel” Fellowship).

\section{References}

1. Maitra A, Hruban RH (2008) Pancreatic cancer. Annu Rev Pathol 3: 157-188.

2. Hidalgo M (2010) Pancreatic cancer. N Engl J Med 362: 1605-1617.

3. Navas C, Hernández-Porras I, Schuhmacher AJ, Sibilia M, Guerra C, et al. (2012) EGF receptor signaling is essential for k-ras oncogene-driven pancreatic ductal adenocarcinoma. Cancer Cell 22: 318-330.

4. Hruban RH, Maitra A, Kern SE, Goggins M (2007) Precursors to pancreatic cancer. Gastroenterol Clin North Am 36: 831-849.

5. Hingorani SR, Petricoin EF, Maitra A, Rajapakse V, King C, et al. (2003) Preinvasive and invasive ductal pancreatic cancer and its early detection in the mouse. Cancer Cell 4: 437-450.

6. Malumbres M, Barbacid M (2003) RAS oncogenes: the first 30 years. Nat Rev Cancer 3: 459-465.

7. Hingorani SR, Wang L, Multani AS, Combs C, Deramaudt TB, et al. (2005) Trp53R172H and KrasG12D cooperate to promote chromosomal instability and widely metastatic pancreatic ductal adenocarcinoma in mice. Cancer Cell 7: 469-483.

8. DeNicola GM, Tuveson DA (2009) RAS in cellular transformation and senescence. Eur J Cancer 45 Suppl 1: 211-216.

9. Guerra C, Schuhmacher AJ, Cañamero M, Grippo PJ, Verdaguer L, et al. (2007) Chronic pancreatitis is essential for induction of pancreatic ductal 
Citation: Hessmann E, Ellenrieder V, Koenig A (2014) NFAT in Pancreatic Carcinogenesis. J Carcinog Mutagen 5: 203. doi:

Page 5 of 5

adenocarcinoma by K-Ras oncogenes in adult mice. Cancer Cell 11 291-302.

10. Guerra C, Collado M, Navas C, Schuhmacher AJ, Hernandez-Porras I, et al. (2011) Pancreatitis-induced inflammation contributes to pancreatic cancer by inhibiting oncogene-induced senescence. Cancer Cell 19:728-739.

11. Mancini M, Toker A (2009) NFAT proteins: emerging roles in cancer progression. Nat Rev Cancer 9: 810-820.

12. Arlt A, Schäfer H, Kalthoff $\mathrm{H}$ (2012) The 'N-factors' in pancreatic cancer: functional relevance of NF- ${ }^{\circ} \mathrm{B}$, NFAT and Nrf2 in pancreatic cancer. Oncogenesis 1: e35.

13. Hogan PG, Chen L, Nardone J, Rao A (2003) Transcriptional regulation by calcium, calcineurin, and NFAT. Genes Dev 17: 2205-2232.

14. Baumgart S, Ellenrieder V, Fernandez-Zapico ME (2013) Oncogenic transcription factors: cornerstones of inflammation-linked pancreatic carcinogenesis. Gut 62: 310-316.

15. Chuvpilo S, Avots A, Berberich-Siebelt F, Glackner J, Fischer C, et al. (1999) Multiple NF-ATc isoforms with individual transcriptional properties are synthesized in T lymphocytes. J Immunol 162: 7294-7301.

16. Chuvpilo S, Zimmer M, Kerstan A, Glöckner J, Avots A, et al. (1999) Alternative polyadenylation events contribute to the induction of NFATc in effector T cells. Immunity 10: 261-269.

17. Fernandez-Zapico ME, Ellenrieder V (2010) NFAT transcription factors, the potion mediating "Dr. Jekill-Mr. Hyde" transformation of the TGFI' ${ }^{2}$ pathway in cancer cells. Cell Cycle 9: 3838-3839.

18. Singh SK, Baumgart S, Singh G, Kanig AO, Reutlinger K, et al. (2011) Disruption of a nuclear NFATc2 protein stabilization loop confers breast and pancreatic cancer growth suppression by zoledronic acid. J Biol Chem 286: 28761-28771.

19. Korc M, Chandrasekar B, Yamanaka Y, Friess H, Buchier M, et al. (1992) Overexpression of the epidermal growth factor receptor in human pancreatic cancer is associated with concomitant increases in the levels of epidermal growth factor and transforming growth factor alpha. J Clin Invest 90: 1352-1360.

20. Ardito CM, Grüner BM, Takeuchi KK, Lubeseder-Martellato C, Teichmann N, et al. (2012) EGF receptor is required for KRAS-induced pancreatic tumorigenesis. Cancer Cell 22: 304-317.

21. Friday BB, Horsley V, Pavlath GK (2000) Calcineurin activity is required for the initiation of skeletal muscle differentiation. J Cell Biol 149: 657-666.

22. Graef IA, Chen F, Chen L, Kuo A, Crabtree GR (2001) Signals transduced by $\mathrm{Ca}(2+) /$ calcineurin and $\mathrm{NFATc} 3 / \mathrm{c} 4$ pattern the developing vasculature. Cell 105: 863-875.

23. Neal JW, Clipstone NA (2003) A constitutively active NFATc1 mutant induces a transformed phenotype in 3T3-L1 fibroblasts. J Biol Chem 278: 17246-17254.

24. Buchholz M, Schatz A, Wagner M, Michl P, Linhart T, et al. (2006) Overexpression of c-myc in pancreatic cancer caused by ectopic activation of NFATcl and the $\mathrm{Ca} 2+/$ calcineurin signaling pathway. EMBO J 25: 3714-3724.

25. Ottenhof NA, de Wilde RF, Maitra A, Hruban RH, Offerhaus GJ (2011) Molecular characteristics of pancreatic ductal adenocarcinoma. Patholog Res Int: 620601.

26. Jones S, Zhang X, Parsons DW, Lin JC, Leary RJ, et al. (2008) Core signaling pathways in human pancreatic cancers revealed by global genomic analyses. Science 321: 1801-1806

27. Köenig A, Linhart $\mathrm{T}$, Schlengemann $\mathrm{K}$, Reutlinger $\mathrm{K}$, Wegele J, et al. (2010) NFAT-induced histone acetylation relay switch promotes c-Myc- dependent growth in pancreatic cancer cells. Gastroenterology 138: 1189-1199.

28. Ellenrieder V (2008) TGFbeta regulated gene expression by Smads and Sp1/KLF-like transcription factors in cancer. Anticancer Res 28: 1531-1539.

29. Singh G, Singh SK, König A, Reutlinger K, Nye MD, et al. (2010) Sequential activation of NFAT and c-Myc transcription factors mediates the TGF-beta switch from a suppressor to a promoter of cancer cell proliferation. J Biol Chem 285: 27241-27250.

30. Hahn SA, Schutte M, Hoque AT, Moskaluk CA, da Costa LT, et al. (1996) DPC4, a candidate tumor suppressor gene at human chromosome 18q21.1. Science 271: 350-353.

31. Baumgart S, Chen NM, Siveke JT, König A, Zhang JS, et al. (2014) Inflammation-induced NFATc1-STAT3 transcription complex promotes pancreatic cancer initiation by KrasG12D. Cancer Discov 4: 688-701.

32. Larsson LG (2011) Oncogene- and tumor suppressor gene-mediated suppression of cellular senescence. Semin Cancer Biol 21: 367-376.

33. Blackburn EH (1994) Telomeres: no end in sight. Cell 77: 621-623.

34. Blackburn EH, Epel ES (2012) Telomeres and adversity: Too toxic to ignore. Nature 490: 169-171.

35. Singh SK, Ellenrieder V (2013) Senescence in pancreatic carcinogenesis: from signalling to chromatin remodelling and epigenetics. Gut 62 1364-1372.

36. Schutte B, Nieland L, van Engeland M, Henfling ME, Meijer L, et al. (1997) The effect of the cyclin-dependent kinase inhibitor olomoucine on cell cycle kinetics. Exp Cell Res 236: 4-15.

37. Baumgart S, Glesel E, Singh G, Chen NM, Reutlinger K, et al. (2012) Restricted heterochromatin formation links NFATc2 repressor activity with growth promotion in pancreatic cancer. Gastroenterology 142:388-398.

38. Griesmann H, Ripka S, Pralle M, Ellenrieder V, Baumgart S, et al. (2013) WNT5A-NFAT signaling mediates resistance to apoptosis in pancreatic cancer. Neoplasia 15: 11-22.

39. Bayne LJ, Beatty GL, Jhala N, Clark CE, Rhim AD, et al. (2012) Tumorderived granulocyte-macrophage colony-stimulating factor regulates myeloid inflammation and $\mathrm{T}$ cell immunity in pancreatic cancer. Cancer Cell 21: 822-835.

40. Pylayeva-Gupta Y, Lee KE, Hajdu CH, Miller G, Bar-Sagi D (2012) Oncogenic Kras-induced GM-CSF production promotes the development of pancreatic neoplasia. Cancer Cell 21: 836-847.

41. Kaufman DB, Shapiro R, Lucey MR, Cherikh WS, T Bustami R, et al. (2004) Immunosuppression: practice and trends. Am J Transplant 4 Suppl 9: 38-53.

42. Dantal J, Soulillou JP (2005) Immunosuppressive drugs and the risk of cancer after organ transplantation. N Engl J Med 352: 1371-1373.

43. McCleary-Wheeler AL, Lomberk GA, Weiss FU, Schneider G, Fabbri M, et al. (2013) Insights into the epigenetic mechanisms controlling pancreatic carcinogenesis. Cancer Lett 328: 212- 221.

44. Zhang JS, Koenig A, Harrison A, Ugolkov AV, Fernandez-Zapico ME, et al. (2011) Mutant K-Ras increases GSK-3 $\hat{I}^{2}$ gene expression via an ETSp300 transcriptional complex in pancreatic cancer. Oncogene 30: 3705-3715.

45. Ougolkov AV, Bilim VN, Billadeau DD (2008) Regulation of pancreatic tumor cell proliferation and chemoresistance by the histone methyltransferase enhancer of zeste homologue 2. Clin Cancer Res 14: 6790-6796. 